\title{
\%
}

\author{
Luis Óscar Moreno García-Cano*
}

\section{LA GEOPOLÍTICA DEL GAS: EL MAGREB Y EL SUMINISTRO ENERGÉTICO EN ESPAÑA}

La geopolítica siempre ha influido en el panorama energético global y, en particular, en el de los combustibles fósiles. El caso arquetípico es el de la crisis energética de los años setenta. No obstante, el contexto actual de tensiones energéticas y volatilidad de los mercados ha vuelto a poner sobre el tapete la relevancia de la geopolítica en el suministro y precio de los combustibles fósiles y, en particular, del gas. Un ejemplo de esto es el caso europeo, donde tanto Argelia como Rusia están privilegiando los gasoductos directos (Medgaz y Nord Stream), frente a aquellos que transitan por terceros países como Marruecos o Ucrania, por motivos e intereses que van más allá de lo económico.

Palabras clave: geopolítica, Magreb, suministro, gasoducto, interconexiones, infrainversión. Clasificación JEL: Q40, F50, F52.

\section{Introducción}

Tras la pandemia, las economías mundiales se han enfrentado de golpe con una dura realidad: la fuerte dependencia que aún existe de los combustibles fósiles y el impacto que la subida de precio de estos últimos tiene sobre los balances de empresas y familias. El petróleo Brent se mueve actualmente por encima de los 80 dólares el barril, su nivel más alto desde 2014, y el gas natural ha subido más de un $500 \%$ en Europa y un $130 \%$ en Estados Unidos en lo que va de 2021. Incluso el casi olvidado carbón se revaloriza un $100 \%$ este año. Esto ha sacado a la luz problemas profundos

\footnotetext{
* Técnico Comercial y Economista del Estado. Versión de noviembre de 2021.

DOI: https://doi.org/10.32796/bice.2021.3141.7318
}

en el sector energético, a medida que el mundo se orienta hacia un sistema energético más limpio, como el aumento de los riesgos geopolíticos y los endebles amortiguadores de seguridad ante problemas de suministro. El mercado de la energía no había visto subidas de precios tan dramáticas desde la crisis del petróleo de los años setenta, cuando el cartel de productores OPEP descubrió que podía mantener en jaque al mundo desarrollado activando y desactivando el suministro.

La idea de escasez de oferta energética parecía ridícula en 2020, cuando la demanda energética mundial se redujo un $5 \%$-el mayor descenso desde la Segunda Guerra Mundial, según Statistical Review of World Energy 2021 (BP, 2021)—, pero a medida que la economía mundial se ha ido reactivando, la demanda energética ha aumentado por encima de la $\triangleright$ 
oferta, reduciendo así las reservas estratégicas peligrosamente. De hecho, los inventarios de petróleo solo alcanzan el $94 \%$ de su nivel habitual; los de gas europeo, el $86 \%$; y los de carbón de la India y China, menos del 50\% (The first big energy shock of the green era, 2021).

En el contexto actual de recuperación poscovid y elevada demanda, los mercados de la energía están siendo especialmente vulnerables a perturbaciones por el lado de la oferta. La primera de ellas es la falta de inversión. Los combustibles fósiles siguen satisfaciendo el $83 \%$ de la demanda de energía primaria (Foulis, 2021), pero las presiones regulatorias y de los propios inversores han hecho que la inversión en combustibles fósiles se desplome un 40\% desde 2015 (International Energy Agency -IEA-, 2020). La falta de inversión y el desplazamiento del modelo de negocio de las grandes empresas privadas de hidrocarburos hacia las energías renovables están provocando un shock de oferta negativo en el sector.

El segundo fenómeno que acecha a la oferta fósil es el geopolítico. Según algunos países OCDE van abandonando la producción de combustibles fósiles, esta se desplaza y se concentra en países con menor necesidad de rendición de cuentas, aumentando así el poder de mercado de estos. El porcentaje de producción de petróleo de la OPEP más Rusia se prevé que pase del 46 actual al 50 o más en 2030 (Foulis, 2021). Además, Rusia es el proveedor del $43,4 \%$ de las importaciones de gas de Europa (Eurostat, 2021) y su influencia aumentará a medida que abra el gasoducto Nord Stream 2 y desarrolle mercados en Asia.

En el caso español, la geopolítica también importa. Según Enagás (2021), Argelia provee el $47 \%$ del gas que entra en España $-y$ el
12\% del gas europeo, según Eurostat (2021) y acaba de cerrar el suministro de gas por uno de los dos gasoductos que conectan los pozos de Hassi R'Mel (Argelia) con España. Al privilegiar Argelia su gasoducto directo a España (Medgaz), se estaría replicando, de alguna manera, lo que sucede entre Rusia y Alemania con otro gasoducto directo: el Nord Stream 2.

\section{Infrainversión en combustibles fósiles}

En los últimos meses hemos visto cortes y racionamiento de suministro eléctrico en China, escasez de combustible en Reino Unido, falta de carbón en India o aumento disparado de los precios del gas y la electricidad en Europa. Esto, en parte, es un efecto colateral del periodo de exceso de oferta (los años de sobreinversión dieron lugar a una mayor disciplina de capital) o del Brexit en el caso británico, pero, sobre todo, es el resultado de las crecientes señales para descarbonizar por parte del regulador (fiscales, regulatorias, de precio del $\mathrm{CO}_{2}$, etc.) y de los fondos de inversión (que son accionistas de las empresas energéticas y que cada vez más invierten por criterios $\mathrm{ESG}^{1}$ ). Este año, el déficit de inversión es una de las principales razones por las que los precios de los combustibles fósiles se han disparado.

Empezando por el petróleo, es una industria que necesita constantes inversiones solo para mantenerse en pie. Una regla general es que las empresas petroleras deben destinar unas cuatro quintas partes de su gasto de capital cada año solo para impedir que se agote su nivel de reservas. Y esta industria solía $D$

Los criterios ESG son factores ambientales, sociales y de gobierno corporativo que se tienen en cuenta a la hora de invertir en una empresa. 
responder a la fuerte demanda y al aumento de los precios invirtiendo en la extracción de más petróleo. Sin embargo, las grandes compañías petroleras del sector privado, especialmente las europeas, están siendo presionadas por sus inversores para que traten sus inversiones en petróleo y gas como si fueran de corto plazo, retrayendo así su capex (gasto de capital). Esto se debe a que sus accionistas consideran que la demanda de combustibles fósiles acabará por alcanzar eventualmente su punto máximo, lo que hará que los proyectos a largo plazo no sean rentables (considerándolos como activos «varados») o prefieran tener participaciones en empresas que apoyen la transición a una energía limpia. Además, en el ámbito legal se está abriendo otro frente, puesto que ya se están produciendo sentencias ${ }^{2}$ que obligan a las empresas petroleras privadas a bajar de forma más acelerada sus emisiones.

El resultado de todo esto es una caída mundial de la inversión en exploración y producción de petróleo y gas, que ha pasado de más de 800.000 millones de dólares en 2014 a apenas unos 400.000 millones actualmente (Don't expect big oil to fix the energy crunch, 2021).

Otro factor que inhibe la inversión es el comportamiento de los países de la OPEP+ ${ }^{3}$. La media década de precios relativamente bajos, que alcanzó su punto álgido con el colapso de los precios al comienzo de la pandemia, vació las arcas estatales de estos países. Con la recuperación de los precios, la prioridad de los Gobiernos no ha sido ampliar la capacidad de

2 Un tribunal neerlandés consideró, en mayo de 2021, que la petrolera Shell «puede» y «debe» adherirse al Acuerdo Climático de París y bajar de forma más acelerada sus emisiones con una reducción neta del $45 \%$ en 2030, en un respaldo sin precedentes a la denuncia de la ONG Amigos de la Tierra (Milieudefensie, en neerlandés).

3 En 2016 los trece países de la OPEP lograron una alianza con un grupo de diez países (liderados por Rusia), a la que denominarían OPEP+. Los diez nuevos miembros son: Rusia, México, Kazajstán, Azerbaiyán, Baréin, Brunéi, Malasia, Omán, Sudán y Sudán del Sur. producción de petróleo o gas, sino apuntalar los presupuestos nacionales. Además, los productores estatales se han mostrado, por el momento, cautelosos, preocupados por la posibilidad de que un nuevo brote de casos de coronavirus pudiera de nuevo afectar a la demanda. En cualquier caso, incluso si el repunte de precios actual acabara por empujar la inversión, harían falta varios años para aumentar significativamente la producción de petróleo y gas (la producción de petróleo tiene un efecto indirecto en la producción de gas natural, ya que este suele ser un subproducto de la perforación de crudo).

La inversión en carbón es la más débil de todas. Incluso en China e India, que planean construir nuevas centrales eléctricas de carbón, el ánimo inversor se ha inclinado en contra del combustible fósil más sucio, tanto por sus emisiones de carbono y su precio en derechos de emisión como por su efecto en la calidad del aire. Sin embargo, la demanda puede aumentar coyunturalmente a medida que China se adentre en un invierno frío e India tenga problemas de suministro.

\section{El caso del gas}

El gas natural desempeña una función vital tanto en el mantenimiento de la estabilidad de la red eléctrica, especialmente en países que dependen de la energía eólica y solar (de carácter fundamentalmente intermitente), como en la transición hacia una economía descarbonizada (por sus menores emisiones relativas respecto de otras fuentes fósiles de energía). Sin embargo, los precios del gas están experimentando un aumento sin precedentes a lo largo de este 2021, que está afectando tanto a la estabilidad de los sistemas eléctricos como $\square$ 
a la propia transición energética. Esto ha sido más evidente en los mercados de gas europeos y asiáticos, ambos grandes importadores de gas natural. En términos interanuales, los precios del gas natural licuado (GNL) JKM ${ }^{4}$ asiático han subido un $450 \%$, mientras que los precios del gas TTF 5 europeo han subido un $500 \%$ (Oxford Economics, 2021b).

Se ha producido una tormenta casi perfecta de factores que impulsan esta evolución extrema de los precios, siendo algunos de ellos de carácter coyuntural (recuperación de la demanda tras la pandemia), pero otros muchos lo son de carácter estructural, como acabamos de explicar.

Además, el invierno especialmente frío del hemisferio norte en 2020/21 y la velocidad del viento más lenta de lo normal en Europa (se ha recurrido más al gas para producir electricidad) provocaron una disminución sensible de los inventarios de gas natural. A los factores climáticos hay que añadir problemas de producción en el mar del Norte que dificultaron también la reposición de existencias a tiempo. Así, las cifras de reservas de gas natural en Europa, publicadas por la IEA (2020), indican que los inventarios continentales están un $16 \%$ por debajo de la media de los cinco últimos años y un $22 \%$ por debajo de lo almacenado hace doce meses. En España, las reservas de gas están, a 25 de octubre de 2021 , al $80,6 \%$, cuando un año antes estaban al 94,8\% (CORES, 2021).

Mientras tanto, la demanda, incluida la energética, ha regresado con un sorprendente dinamismo a medida que la pandemia ha ido

\footnotetext{
4 Platts JKM es un precio de referencia del gas natural licuado (GNL) para cargas físicas al contado. Se toma como referencia en las operaciones al contado, las licitaciones y los contratos a corto, medio y largo plazo tanto en el noreste de Asia como en el resto del mundo.

5 TTF (Title Transfer Facility) es un punto de comercio virtual de gas natural en Países Bajos que sirve de referencia en el continente europeo. Este punto de negociación ofrece a una serie de operadores de este país la posibilidad de negociar con futuros, con productos físicos y con intercambios.
}

remitiendo. $Y$ este dinamismo es probable que se mantenga en el medio plazo porque se calcula que las importaciones de gas natural licuado de China se duplicarán de aquí a 2030, según Bernstein (como se citó en Don’t expect big oil to fix the energy crunch, 2021). Pero si no se invierte en nuevos proyectos, la capacidad mundial de GNL será un $14 \%$ inferior a la necesaria para entonces, lo que ahondará en el desajuste entre oferta y demanda (Bernstein, como se citó en Don't expect big oil to fix the energy crunch, 2021).

Rusia y Argelia son los únicos proveedores importantes de gas natural de Europa que han aumentado el suministro en el primer semestre de 2021 en relación con el primer semestre de 2020. Sin embargo, la producción de gas natural de EE. UU. se ha visto afectada por problemas este año. La tormenta de febrero en Texas, el ciberataque de Colonial Pipeline en mayo y el huracán Ida en septiembre tuvieron un impacto notable en la producción estadounidense. Existe un problema de déficit de capacidad: antes de la pandemia, EE. UU. tenía 790 pozos de gas o petróleo activos y, en julio de este año, solo 251 (Aparicio, 2021). Ponerlos de nuevo en marcha tiene sus dificultades. Para el fracking, el costo de perforar un pozo y ponerlo en producción ha subido alrededor de un $2,5 \%$ en cada uno de los tres primeros trimestres de 2021, y se espera otro aumento del $3 \%$ antes de los tres últimos meses de 2021, según la consultora Spears \& Associates. Tampoco hay suficientes trabajadores cualificados para cubrir todas las vacantes en el sector petrolero, y los salarios han subido mucho, según los ejecutivos de servicios petroleros encuestados por el Banco de la Reserva Federal de Dallas. De este modo, la capacidad de EE. UU. para enviar GNL a los mercados europeos y asiáticos se está viendo limitada. 


\subsection{La geopolítica del gas}

\section{El gas natural licuado (GNL)}

Las importantes reservas de gas natural que existen en nuestro planeta, a veces, están situadas en zonas remotas, carecen de demanda local y el transporte del gas a través de gasoductos, en ocasiones, no es rentable. Pero los avances tecnológicos de los últimos años han hecho técnica y económicamente viable el transporte del gas en fase líquida (enfriado a $-160 \stackrel{\circ}{\circ})$ mediante buques metaneros. El gas transportado en fase líquida se conoce como gas natural licuado (GNL).

La mayor parte del GNL está casi asegurado en contratos a largo plazo y tiene como destino Asia. Estos contratos de largo plazo suelen estar vinculados al petróleo y suponen el grueso de las importaciones/exportaciones de gas natural. Sin embargo, la escasez de gas está dando protagonismo a las compras al contado y a los contratos a corto plazo, que sirven para equilibrar los desajustes por un aumento inesperado del consumo. El hambre por el gas al contado, ante los bajos niveles de inventarios, es lo que está disparando el precio de este combustible.

Además, un verano caluroso ha contribuido al auge de la demanda de gas en Asia en el año en curso. La región representa casi tres cuartas partes de las importaciones mundiales de GNL, según AllianceBernstein (como se citó en Natural-gas prices are spiking around the world, 2021). En el primer semestre de 2021, su generación de energía se disparó un 16\% respecto al año anterior. Tres quintas partes de la energía de China se generan con carbón y una quinta parte procede de la energía hidroeléctrica. Pero la generación hidroeléctrica ha sido baja debido a la sequía, y la demanda de carbón se ha reducido motivada por las políticas de transición energética (como, por ejemplo, la sustitución de las calderas de carbón por otras de gas). La inversión en la extracción de carbón también ha sido baja. Todo esto ha supuesto una mayor dependencia del gas natural en el país. Así, en el primer semestre del año 2021, la generación de gas creció más rápido que la de carbón o la hidroeléctrica y las importaciones chinas de GNL crecieron un $26 \%$ respecto al año anterior. De hecho, la IEA prevé que China represente el $30 \%$ del aumento de la demanda mundial de gas de aquí a 2024.

Además, Japón, India, Corea del Sur o Taiwán también están comprando, guiados por el pánico, ante la posibilidad de un invierno frío en 2021-2022. Esto está incrementando la competencia por la fracción de la oferta que se comercializa libremente en el mercado al contado y no está vinculada a contratos a largo plazo. Mientras tanto, la sequía en América Latina, que obtiene la mitad de su energía de la hidroeléctrica, ha aumentado la necesidad de gas, y la demanda de GNL en la región casi se ha duplicado en el último año.

Con la succión de GNL hacia Asia y América Latina, ha quedado menos para los compradores europeos. De este modo, las importaciones de GNL en Europa son en 2021 un 20\% inferiores a las de 2020. Además, la producción de gas también ha disminuido en Gran Bretaña y Países Bajos. Los analistas esperaban que la rusa Gazprom, que suministra un tercio del gas europeo, compensara la diferencia, pero, aunque este año ha cumplido todos sus contratos de gas a largo plazo con Europa, no ha vendido, por el momento, suficiente gas en el mercado al contado. Algunos analistas hablan de cierta preferencia por la aceleración de la puesta en marcha del Nord Stream 2 y de la reposición de sus propias reservas antes de liberar más gas a Europa. 


\section{Los gasoductos}

El otro sistema de transporte de gas entre dos puntos es el gasoducto, que puede estar enterrado en la superficie terrestre o en el fondo de los océanos. La capacidad de transporte de los gasoductos depende de la diferencia de presión entre sus extremos y de su diámetro.

Los gasoductos proveen una seguridad de suministro y una estabilidad en precio que no ofrece el mercado del GNL, que hoy en día es extremadamente competitivo (las cargas de gas al contado se entregan allá donde haya mejor netback - retorno respecto a los costes de transportar y extraer el gas-). Este año, para evitar la escasez de buques, que ya se sufrió el invierno pasado, y los costes sin precedentes para hacerse con GNL al contado, los importadores y comercializadoras se han adelantado y han estado reservando barcos desde principios de este año (lo que podría agudizar aún más la competencia por los pocos barcos que queden libres en épocas punta de demanda).

En el caso europeo, después de años de hablar sobre la diversificación del suministro de gas, pero haciendo poco para promoverla, ahora no se encuentra la forma de obligar a Gazprom a enviar más gas. Todo esto con la vista puesta en una pronta aprobación del Nord Stream 2. De todos modos, Bank of America considera que el plan de Gazprom de llenar el $50 \%$ de la capacidad de Nord Stream 2 en este último trimestre del año solo aliviaría aproximadamente la mitad del déficit de inventario estacional de Europa.

Por otro lado, más allá de Rusia, Noruega no tiene capacidad por sí sola para bombear todo el gas que necesitan sus vecinos europeos (Noruega provee el $20 \%$ del gas europeo).

Para acercarnos al caso español, es de interés ver lo que sucede con el gasoducto Nord
Stream 2, que provee gas directamente desde Rusia a Alemania. Se trata de un caso espejo al de nuestro gasoducto hispano-argelino, Medgaz, que viene directamente desde Argelia hasta España sin pasar por Marruecos.

Rusia suministra gas al centro y norte de Europa mediante diversos gasoductos que atraviesan Bielorrusia (el 20\% del gas transita por territorio bielorruso) y Ucrania (cerca de un tercio del gas transita por territorio ucraniano) antes de entrar en territorio comunitario. También suministra de manera directa a Alemania, a través del mar Báltico, mediante los gasoductos Nord Stream 1 y Nord Stream $2^{6}$. Este último, el Nord Stream 2, que ha costado 9.500 millones de euros, duplicará la capacidad de transporte de gas natural de Rusia a Alemania cuando esté terminado en 2021. Durante años, este gasoducto ha provocado divisiones entre Europa y EE. UU., así como dentro de Europa. Alemania lo apoya enérgicamente, mientras que EE. UU. se opone, argumentando que otorga demasiado poder de mercado (y por tanto geopolítico) a Rusia. Sin embargo, las enormes tuberías se han colocado a lo largo del Báltico y, salvo problemas de última hora, el gas empezará a fluir por ellas en breve?

Como se señalaba anteriormente, gran parte del gas que fluye de Rusia a Europa pasa actualmente por Ucrania, lo que hace que el país gane unos 2.000 millones de dólares al año en concepto de tasas de tránsito $\square$

\footnotetext{
6 Nord Stream 1 tiene una capacidad anual total de $55 \mathrm{bcm}$ de gas, y se espera que la adición de Nord Stream 2 duplique esta capacidad a un total de $110 \mathrm{bcm}$.

7 El regulador alemán, la Agencia Federal de Redes, tiene hasta el 8 de enero de 2022 para recomendar si certificar o no el gasoducto. Después del visto bueno de tres miembros del comité independiente de la agencia, esta debe pasar a la Comisión Europea, que cuenta con un plazo de dos meses para responder. Entre otras condiciones, las reglas de competencia europeas requieren que los propietarios de los gasoductos sean diferentes de los proveedores de gas que operan en la red. En este caso, la subsidiaria de Gazprom, Nord Stream AG, es la gestora de estas tuberías, pero el Nord Stream 1 ha estado exento de las reglas de separación desde su inauguración.
} 
(Ukrinform, 2020). Se trata de una parte importante del presupuesto estatal. Rusia ha prometido mantener el flujo de gas a través de Ucrania hasta, al menos, finales de 2024, pero el Gobierno de Kiev teme que, una vez completado el Nord Stream 2, Rusia desvíe sus suministros de gas directamente a Alemania. Para disipar estos temores, Merkel insistió, en la víspera de su viaje a Estados Unidos, en que «Nord Stream 2 no sustituye al suministro prometido a través de Ucrania».

El Gobierno de Obama, en sus esfuerzos por aislar a Putin, se quejó de que el Nord Stream 2 -como el Nord Stream original que se inauguró en 2011- haría a Europa aún más dependiente del gas ruso —el $43 \%$ de las importaciones de gas natural de la UE procedían de Rusia en 2020 (Eurostat, 2021) - y aislaría a Ucrania. Rusia, por su parte, argumentó que la preocupación de EE. UU. por Ucrania era una pista falsa; a sus ojos, EE. UU. quería detener el acuerdo para poder enviar más de su propio GNL a Europa. En apoyo de Ucrania, y para castigar al Kremlin por la anexión de Crimea, el Congreso de EE. UU. trató de anular el gasoducto imponiendo sanciones a algunas empresas implicadas en el Nord Stream 2. Biden ha adoptado un enfoque más pragmático. Su Administración insiste en que EE. UU. sigue oponiéndose al proyecto Nord Stream 2 por motivos de seguridad. Sin embargo, en mayo, señalando que el oleoducto está «casi completamente terminado», renunció a las sanciones estadounidenses contra las empresas detrás del gasoducto, ya que serían «contraproducentes» para la relación transatlántica.

El Gobierno alemán ha confirmado que se han concedido todos los permisos de construcción, y la empresa que está detrás del gasoducto espera que se ponga en marcha este mismo año. El consorcio que financia el proyecto, formado por Gazprom y sus cinco patrocinadores (las alemanas Uniper y Wintershall, la austriaca OMV, la francesa Engie y la angloneerlandesa Royal Dutch Shell), se ha mantenido firme. Los gigantes industriales alemanes, como BASF, consideran esencial asegurarse el gas ruso para conseguir la seguridad de suministro y estabilidad en el precio para competir con sus rivales estadounidenses.

\section{El caso español: los gasoductos con Argelia y Marruecos}

España, con datos anualizados a septiembre de 2021, recibe por gasoducto el 50,6\% del gas (el resto es GNL) y, a excepción de Francia (por donde entra un 6,8\%), prácticamente todo el gas por gasoducto procede de Argelia.

Según datos del Boletín Estadístico de Enagás (2021), las entradas de gas natural a España procedentes de Argelia — sumando el gas natural que llega por gasoducto y el gas natural licuado que viene por barco-fueron el $51 \%$ en 2018 , el $33 \%$ en 2019 , el $29 \%$ en 2020 y el $47 \%$ en lo que va de 2021. Argelia es, por tanto, el principal proveedor de gas de España y, en el último año, ha aumentado su posición preeminente en la provisión de gas.

España importa gas de Argelia a través de dos gasoductos: el Medgaz, que va de Beni Saf (Argelia) hasta la costa de Almería por el Mediterráneo, por donde entra el 20,8\% del gas en España; y el Magreb-Europa (GME), que atraviesa Marruecos y, desde Tánger, cruza el Estrecho hasta Tarifa, por donde entra el 21,9\% del gas en España. Los dos gasoductos se han utilizado indistintamente en función de las necesidades, del destino final del gas y del precio del uso de las infraestructuras, lo que otorga $\triangleright$ 
flexibilidad y estabilidad al sistema gasista español.

EI GME es un gasoducto de unos 1.400 kilómetros de longitud, de los que 540 discurren en territorio marroquí, antes de cruzar el estrecho de Gibraltar y terminar en Córdoba. El gasoducto parte de un acuerdo intergubernamental firmado el 30 de abril de 1991 entre España, Argelia y Marruecos, y fue inaugurado en 1996. Tiene la capacidad de transportar cada año 13,5 bcm (billion cubic metres o mil millones de metros cúbicos) a España y Portugal. El GME fue un proyecto original de la antigua Gas Natural; de hecho, aún se le conoce por el nombre del presidente de la compañía en la época: Durán Farrell. Para su construcción y ejecución, el proyecto se dividió en cuatro tramos, gestionados por Sonatrach (tramo argelino), Europe Maghreb Pipeline Limited (EMPL) y Metragaz (Marruecos), Transgas (Portugal) y Enagás (España), entonces propiedad de Gas Natural.

El otro gasoducto, el Medgaz, inaugurado en 2011 y que conecta los yacimientos de gas de Hassi R'mel con la red española en la ciudad de Almería, tiene una capacidad actual de $8 \mathrm{bcm}$. No obstante, en este momento, se está trabajando en un proyecto para aumentar la capacidad de esta infraestructura, que a partir del próximo mes de diciembre, y siempre que la ampliación se concluya en plazo, pasaría a transportar anualmente unos $10 \mathrm{bcm}$ de gas. Esta inversión, estimada en unos 73 millones de euros, ha corrido a cargo de las compañías propietarias de la infraestructura, la argelina Sonatrach y la española Naturgy, que a finales de 2019 amplió su participación estratégica en el gasoducto desde un $15 \%$ hasta un $49 \%$. Este incremento en la participación se llevó a cabo con la compra a Mubadala (Cepsa) de un $34,05 \%$ del capital, a través de un vehículo de propósito especial (SPV). Posteriormente, en marzo de 2020, Naturgy cerró con BlackRock su entrada en la SPV.

Con la construcción de Medgaz, Argelia evitaba, potencialmente hasta ahora, la servidumbre de paso que tenía con Marruecos con el GME. Una situación que, como hemos mencionado antes, se podría equiparar a lo que ocurre con el Nord Stream 2 entre Rusia y Ucrania.

Respecto del suministro, cabe señalar que, en los doce meses transcurridos entre septiembre de 2020 y agosto de 2021, Argelia suministró a España unos $15 \mathrm{bcm}$, equivalentes al $46 \%$ de nuestras necesidades de gas (Enagás, 2021). De estos $15 \mathrm{bcm}$, seis fueron suministrados por el gasoducto que atraviesa Marruecos, ocho por el gasoducto que une directamente la costa argelina con Almería y el bcm restante fue suministrado en barcos, en forma de gas natural licuado.

El pasado 24 de agosto, en plena escalada de los precios energéticos, el ministro de Exteriores argelino, Ramtane Lamamra, anunció la ruptura de relaciones diplomáticas con Marruecos después de varias semanas en las que había ido subiendo la tensión entre ambos países. Tan solo tres días más tarde tomó cuerpo la primera consecuencia práctica de la crisis bilateral entre las dos potencias del Magreb: el ministro de Energía argelino, Mohamed Arkab, dejó entrever en declaraciones públicas que su Gobierno, tras dos años de negociaciones, no renovaría el acuerdo a tres bandas que permite la llegada a la Península de gas argelino a través del GME. Finalmente, el 31 de octubre, el presidente de Argelia, Abdelmayid Tebún, confirmó oficialmente la no renovación del contrato con Marruecos. En esa fecha caducó la concesión que se había otorgado a la empresa Europe Maghreb Pipeline Limited (EMPL), de la $\triangleright$ 
que eran accionistas la española Naturgy $(77,2 \%)$ y la portuguesa Galp Energia $(22,8 \%)$, y que gestionaba el tramo marroquí del GME.

Esto no significa que el gasoducto quede inservible, como vía de tránsito del gas argelino hasta la península ibérica, para siempre. Podría haber un acuerdo bilateral o trilateral más adelante. No obstante, Argelia ha prohibido recientemente a los aviones marroquíes — civiles y militares - entrar en su espacio aéreo, y su ministro Lamamra ha acusado a Rabat de un acto de «terrorismo de Estado» por la muerte de tres camioneros por un ataque con dron en su camino de Nuakchot, capital de Mauritania, a Uargala, en Argelia. Esta reciente escalada en la tensión bilateral no facilitará en el corto plazo que Argelia permita el paso del gas argelino por el GME.

$Y$ es que el principal perdedor del parón en el suministro por el GME es Marruecos, que percibe un peaje del $7 \%$ del valor del gas exportado por el gasoducto. En 2019, esta cifra no llegó a los 50 millones de euros a causa de la caída en el precio de los combustibles, pero en 2014 (con precios más cercanos a los actuales) se llegó a los 200 millones de euros. Además, el GME proporciona gas al mercado marroquí (800 millones de metros cúbicos), a un precio por debajo de mercado, para que las plantas de ciclo combinado de Ain Beni Mathar y Tahaddart (gestionadas por Abengoa y Ende$\mathrm{sa}^{8}$, respectivamente), con una capacidad de $830 \mathrm{MW}$, generen entre un $9 \%$ y un $12 \%$ de la electricidad que consume el reino alauí.

Dado que en Marruecos el plan para crear una planta de regasificación, gasoductos y nuevos ciclos combinados (proyecto conocido como «Gas to Power», que tenía previstas

8 Endesa cuenta con un $20 \%$ de la central de ciclo combinado de Tahaddart. La propiedad la comparte con Siemens y la ONEE (Oficina Nacional de Electricidad y Agua de Marruecos). inversiones por valor de 4.600 millones de dólares) no llegó a concretarse, y ante el temor a quedarse sin suministro de gas a corto plazo, es probable que el país alauí pretenda relanzar un viejo proyecto: el de hacer reversible el GME para poder recibir gas desde España. De esta manera, Marruecos pasaría de exportar gas a la Península a importarlo. Este contraflujo de gas, que en España abordaría el gestor del sistema gasístico -Enagás-, es posible técnicamente y está articulado en la regulación, si bien podría tener un coste político con Argelia (para evitar, al menos parcialmente, este coste, se podría exportar gas por el tubo que fuera directamente importado de un tercer país, probablemente del golfo Pérsico). Requeriría de una inversión del gestor cuyo montante dependería del volumen a transportar y la fijación de un peaje.

Otra posible consecuencia del cierre a corto plazo sería la utilización a pleno rendimiento de las centrales de carbón de Jorf Lasfar, de 2.000 MW, y Safi, de 1.400 MW (las dos centrales ya están funcionando al límite); de la central de fueloil de Mohammedia, de $300 \mathrm{MW}$; y el aumento de la demanda marroquí de electricidad vía las dos interconexiones eléctricas que unen España con Marruecos y que son copropiedad de la Red Eléctrica de España (REE) y la ONEE. Esto podría tener un efecto sobre el precio, probablemente poco significativo dado que la capacidad de intercambio de los cables es de $900 \mathrm{MW}$ y el sistema español tiene una capacidad de 110 GW, y otro quizás más relevante sobre la opinión pública. España es, a día de hoy, en un contexto de alta demanda, netamente exportadora de electricidad a Marruecos (en los dos últimos meses, del total de flujo eléctrico que pasó por los cables, España exportó entre un $86 \%$ y un $63 \%$ ), pero la operadora eléctrica marroquí (ONEE) no paga $\triangleright$ 
nuestros impuestos de producción (luego repercutidos sobre los consumidores y la industria) y su industria no está en el EU ETS (emissions trading system), por lo que no paga derechos de emisión.

En definitiva, todas las opciones para Marruecos son más costosas que el estatus que mantenía hasta el 31 de octubre de 2021. Por ello, el Gobierno marroquí está contemplando, nuevamente (tras el fallido Gas to Power), la posibilidad de construir una unidad flotante para almacenar y regasificar el gas natural licuado. Así, recientemente, el Ministerio de Transición Energética marroquí ha lanzado una licitación para proponer un sistema que permita la recepción, el almacenaje y la regasificación del combustible, cuya infraestructura podría ser localizada en uno de los siguientes puertos: Nador West Med, Kénitra Atlantique, Mohamedia o Jorf Lasfar. Este proyecto debe responder a las necesidades del mercado, estimadas en 1,1 bcm, de los cuales 0,6 bcm estarán destinados a la industria en el horizonte de 2025. No obstante, este proyecto (por los plazos para su puesta en marcha) no resolverá los problemas de seguridad de suministro de manera inmediata.

Aunque, en menor grado, la propia Argelia sale también perjudicada de la no renovación del acuerdo sobre el GME. A partir de noviembre, su capacidad de exportación por vía terrestre se reducirá de $16 \mathrm{bcm}$ a solo $8 \mathrm{bcm}, \mathrm{y}$ luego, a $10 \mathrm{bcm}$ a partir de enero, si se cumplen los plazos de la ampliación del Medgaz.

España, por su parte, perderá estabilidad, flexibilidad y seguridad de suministro al ver cómo su capacidad de abastecimiento por gasoducto se reduce a más de la mitad (al perder hasta 13,5 bcm sobre un total máximo de capacidad de 21,5 bcm). Dependerá, así, de una sola vía de entrada por gasoducto, perdiendo una vía alternativa ante un eventual problema técnico en el Medgaz. La pérdida de capacidad de suministro por vías submarina y terrestre podrá compensarse parcialmente con la importación de GNL. En concreto, supuesta la ampliación de Medgaz y su entrada en funcionamiento en plazo, España tendría que importar unos 4 bcm de GNL adicionales para mantener el nivel anual de suministro de gas argelino. La forma de trasladar esos cuatro millones de metros cúbicos que España todavía necesitaría para cubrir la demanda actual sería en forma de gas natural licuado (GNL) mediante barcos metaneros. En concreto, se necesitarían unos 48 buques para transportar los $4 \mathrm{bcm}$ restantes (cada bcm perdido equivale a unos 12 metaneros). El problema es que el GNL es un producto más caro y pertenece a un mercado muy disputado actualmente.

El elevado precio de importación de GNL se debe al disparado coste del flete y a que para transportar el gas, primero, hay que licuarlo para trasladarlo en barco y, después, regasificarlo cuando llegue a España. Mientras bombear un bcm por el gasoducto cuesta alrededor de 48-50 millones de dólares, suministrar esa misma cantidad fletando barcos supone unos 118-120 millones de euros (Fernández-Cuesta, 2021). Más del doble.

Argelia exportó, el año pasado, un total de $15 \mathrm{bcm}$ de GNL, y sus principales clientes fueron Turquía (38\%), Francia $(29 \%)$ e Italia (19\%). Nuestra cuota fue testimonial (3\%), ya que la doble conexión por tubo hacía innecesaria la importación por barco. Sobre el papel es factible, aunque no será fácil, absorber algo más del $30 \%$ de las exportaciones argelinas de gas natural licuado. Será necesario que la voluntad política del Gobierno argelino se mantenga, que haya metaneros suficientes (hay un total de 642 metaneros en el mundo) y que $\triangleright$ 
los compromisos contractuales adquiridos por Sonatrach lo permitan.

También es posible importar GNL de otros países exportadores para sustituir el gas argelino que entraba por gasoducto. El problema es que en momentos punta de demanda, como, por ejemplo, en situaciones climáticas adversas, la competencia con el resto del mundo puede ser muy intensa, con el efecto que esto puede tener sobre el suministro y, en especial, sobre el precio del flete. Los precios de los fletes han experimentado un importante incremento debido al desajuste entre la oferta de metaneros disponibles y el importante aumento de la demanda asiática de gas ${ }^{9}$. Esto ha hecho que se hayan llegado a desviar, durante el invierno de 2020, barcos que iban a descargar en España hacia Asia para aprovechar el diferencial de precios. Esto no tiene por qué suceder con los metaneros de Argelia o de otros países, pero sí muestra la presión competitiva a la que este mercado está sometido.

Finalmente, la posible inutilización del gasoducto también tiene un coste político sobre la región en su conjunto, que perdería un proyecto integrador y estabilizador, impulsado por España, y que ha funcionado con éxito al margen del cierre de fronteras desde 1994. Argelia y Marruecos mantienen una relación difícil desde que lograron su independencia. El conflicto de fondo siempre ha sido el Sáhara Occidental y la situación entre los dos países se ha agravado en el último año, especialmente tras la ruptura del alto el fuego decretada por el Frente Polisario en noviembre de 2020 y el reconocimiento, en diciembre de 2020 , de la soberanía marroquí sobre el Sáhara Occidental por parte de la Administración Trump.

\footnotetext{
9 Al cierre de 2020, había 642 embarcaciones en todo el planeta habilitadas para transportar GNL. En el informe «Visión del mercado del GNL a 2025", la consultora Deloitte subraya el peso del transporte, ya que, ante una escasez de barcos disponibles, los picos de precio podrían superar los 100.000 dólares.
}

\section{Conclusiones}

Parece que el mercado del gas seguirá dominado por la volatilidad y las tensiones geopolíticas durante un tiempo prolongado.

Uno, porque la oferta de gas será más inelástica al estar constreñida por el compromiso de las principales economías y empresas de reducción de sus inversiones en energías fósiles. De hecho, las empresas de petróleo y gas han disminuido ya su esfuerzo upstream (aguas arriba) en un 60\% desde 2014, según Rystad Energy (como se citó en Offshore, 2021), y en España Repsol ha reducido su actividad de exploración en un $300 \%$ desde 2008 , y para el periodo 2021-2025 la inversión anual prevista para nuevos sondeos exploratorios se sitúa en 150 millones de euros, cuando en 2014 fue de 1.800 millones de euros.

Dos, por la concentración del suministro y el efecto de la geopolítica. En Europa, Rusia abastece el $43 \%$ del total del gas consumido, ostenta las reservas más importantes del planeta y es prácticamente el único productor que puede modular la extracción en función de la situación del mercado. Además, el tránsito del gas ruso por distintos países de la vecindad oriental, entre ellos Ucrania o Bielorrusia, añade incertidumbre a la provisión en un contexto regional de conflictos armados latentes (entre Rusia y Ucrania en la región ucraniana del Donbas) y amenazas híbridas (presión de Bielorrusia a la Unión Europea con refugiados y migrantes en la frontera con Polonia) ${ }^{10}$. En el caso español, Argelia suministra el $46 \%$ del $\triangleright$

10 El jueves 11 de noviembre, el presidente bielorruso, Alexander Lukashenko, amenazó a Europa con detener el tránsito de gas natural ruso a través de su país, en medio del conflicto por los migrantes y refugiados en la frontera entre Bielorrusia y la UE, y en el contexto de una explosión de precios en el mercado europeo de gas ante la llegada del invierno. El presidente ruso, Vladimir Putin, dijo al respecto que esperaba que no se violara el acuerdo de tránsito bilateral existente. 
total de la provisión de gas, y las tensiones bilaterales con Marruecos, como se ha explicado anteriormente, están creciendo en los últimos meses.

Tres, por el creciente consumo de gas en China. Como se ha descrito a lo largo del artículo, el gas se ha convertido en una energía puente imprescindible para cumplir con los compromisos medioambientales chinos (alcanzar el pico de emisiones antes de 2030, reducir la intensidad de emisiones de la economía china para entonces en un $65 \%$ con respecto a los niveles de 2005, que las renovables representen un $25 \%$ de su cesta energética para 2030 y aumentar su reforestación).

Y cuatro, por la insuficiencia de la inversión acumulada en energías renovables, algo que limita las posibilidades de sustitución de las energías fósiles. La Agencia Internacional de la Energía estima que será necesario invertir el equivalente del $2 \%$ del PIB mundial de aquí a 2030 para producir ese efecto sustitución. Además, algunos investigadores anuncian ya nuevos cuellos de botella para la extracción de determinados suministros críticos para la transición energética, como pueden ser el polisilicio, el litio o el coltán. La geopolítica energética, como ha destacado la IEA (2020), se asocia tradicionalmente al gas y al petróleo, pero se está demostrando también que las tecnologías de energía limpia no son inmunes a los peligros geopolíticos.

En definitiva, en este contexto geoeconómico de tensiones y volatilidad en el mercado energético, habrá que tener muy en cuenta los nuevos retos para la seguridad del suministro que aparecen en esta transición energética y tratar de ofrecer soluciones adecuadas para contrarrestarlos.

En primer lugar, se podrían aumentar los colchones de seguridad para absorber la eventual escasez y hacer frente a la intermitencia de la energía renovable. Los Estados deberían contar con más reservas y capacidad de almacenamiento, al igual que los bancos tienen colchones de capital. La Unión Europea ya cuenta con un sistema de reservas de emergencia para el petróleo, que nació al calor de la crisis del sector en los años setenta del siglo pasado, pero no para el gas. Además, un mecanismo de compra conjunta podría servir para adquirir este recurso cuando el precio estuviera bajo con el fin de liberarlo en periodos alcistas, reduciendo así el poder de mercado de los oferentes. Pero existen interrogantes acerca de la capacidad real de almacenaje de los socios comunitarios: la interconexión energética entre países, el precio que tendría mantener las reservas y quién se haría cargo de esa factura. Para la tarea de almacenaje se suelen usar, entre otros espacios, minas de sal o yacimientos agotados, y no todos los Estados miembros cuentan con esta capacidad.

Por otro lado, la idea de separar el consumo de la producción en el tiempo - utilizando baterías gigantes u otro tipo de almacenamiento- ha recibido mucha atención por parte de los distintos actores involucrados. Pero, a día de hoy, esto es poco realista a escala. Por eso, la idea de separar ambas cosas en el espacio está ganando terreno. Para ello, es necesario reforzar las conexiones internacionales que unen las redes nacionales, tanto en el interior de la Unión Europea como con nuestros principales socios energéticos, especialmente los de la vecindad sur en el caso español.

Las interconexiones internacionales generan una serie de ventajas en los países conectados, fundamentalmente: capacidad de respaldo ante la intermitencia de las renovables y mayor seguridad de suministro. Sirva de ejemplo cómo, en enero de 2009, cuando un $\triangleright$ 
fuerte vendaval afectó a las instalaciones españolas y francesas en la zona de los Pirineos, se hizo necesario el apoyo del sistema eléctrico español durante varios días para asegurar el suministro de energía en la región francesa de Perpiñán en condiciones de seguridad.

No obstante, España tiene una capacidad muy limitada de interconexión eléctrica con la Unión Europea (del 6\% y 6.000 MW), muy lejos del objetivo del $15 \%$ que la Comisión ha marcado como meta para cada Estado miembro en 2030. España cuenta también con dos conexiones eléctricas con Marruecos con una capacidad comercial en conjunto de $1.500 \mathrm{MW}$ (REE, 2019). En lo que respecta a la conexión gasista con Europa, aunque contamos con dos gasoductos que nos conectan con Francia y otros dos con Portugal, las cantidades de gas que llegan por ellos son muy bajas ${ }^{11}$.

Por tanto, para cumplir con los objetivos de seguridad y respaldo, anteriormente mencionados, se deberían reforzar las conexiones eléctricas y gasistas con el resto de Europa y con la vecindad sur. En particular, se debería desarrollar una primera interconexión eléctrica con Argelia, así como reforzar las dos existentes con Marruecos (ya hay un compromiso en este sentido que salió de la visita de Estado de los Reyes a Rabat en 2019). No obstante, esto debería ir enmarcado en un proceso más amplio de cooperación e integración económica y energética mayor con la UE, que apoyara financieramente nuevas inversiones en interconexiones, redes de alta tensión y capacidad de energías renovables (esto podría tener encaje en el marco del Global Europa, previamente conocido como

11 Hasta 2019 hubo un proyecto importante para incrementar la capacidad de interconexión gasista con Europa: el MidCat. Este proyecto de gasoducto iba a discurrir entre Martorell (Barcelona) y Barbaira (Languedoc-Rossellón, Francia), a través de los Pirineos, pero tanto la Unión Europea como los reguladores de España y Francia (CNMC y CRE) dieron carpetazo al proyecto en 2019. El proyecto había nacido en 2005 con una inversión prevista de 3.000 millones de euros.
Neighbourhood, Development and International Cooperation Instrument, que es la principal herramienta financiera de la cooperación financiera europea para el periodo 2021-2027, así como en la nueva estrategia de conectividad europea denominada «Global Gateway») $)^{12}$. En este marco, se debería también facilitar la integración de los países de la ribera sur del Mediterráneo en el EU ETS para evitar fenómenos de carbon leakage y entrar en discusiones sobre mecanismos de ajuste en frontera como el CBAM (Carbon Border Adjustment Mechanism). Si esto no es posible en el plano regional, es necesario avanzar bilateralmente en la cooperación con ambos países.

A nivel bilateral, igual que Argelia acompaña a España en su transición ecológica ofreciendo seguridad de suministro y un respaldo energético, España debe tener presente también la transición que debe hacer Argelia hacia las energías renovables, apoyando la investigación y cooperando con el país vecino en hidrógeno, almacenamiento e interconexión de las redes eléctricas. Esta cooperación e integración energética ejercería de amortiguador contribuyendo a reducir así los riesgos geopolíticos que acechan al suministro. La zona está recibiendo un mayor interés en el plano energético bilateral y, a modo de ejemplo, es interesante señalar que Reino Unido está estudiando la viabilidad de tirar un cable de $3.800 \mathrm{~km}$ que uniría su isla con los campos solares de Marruecos ${ }^{13}$.

Por otro lado, al igual que está haciendo Alemania con Nord Stream 2, se debería $\triangleright$

12 El 15 de septiembre de 2021, la Comisión Europea anunció que la Unión Europea pondría en marcha una gran estrategia de conectividad denominada "Global Gateway» como alternativa a la iniciativa de la Ruta de la Seda china.

13 La sociedad británica Xlinks prevé levantar una central eléctrica, solar y eólica en la región marroquí de Guelmim-Oued Noun, en el sur del país, que aspira a convertirse en el mayor cable eléctrico submarino del mundo. Sus promotores esperan que el cable comience a instalarse en 2025 y terminar el proyecto entre 2027 y 2029. El proyecto tendría un coste global de 22.000 millones de dólares. 
valorar la expansión de capacidad del Medgaz más allá de la limitada ampliación actual de 2 bcm. Para garantizar la seguridad y la estabilidad de suministro a un precio estable se debería doblar la capacidad inicial del gasoducto hasta los $16 \mathrm{bcm}$. En todo caso, habrá que seguir insistiendo en conseguir el paso de gas por el GME, actuando como bróker entre las partes (Argelia y Marruecos). De nuevo, el refuerzo de las interconexiones con la Unión Europea (una demanda de los países de la vecindad sur) puede ayudar a convencer a las partes de que mantengan, e incluso incrementen, los flujos de gas. En todo caso, hay que entender que cualquier estrategia con los países del Magreb deberá ser de largo plazo. Se requiere tiempo, persistencia, presencia y evitar los virajes.

Finalmente, habría que revisar también el mix energético para reducir la fuerte dependencia exterior (78\% en España) y reforzar la seguridad de suministro, sobre todo, teniendo en cuenta que se espera que en Argelia el crecimiento de la población aumente la demanda local de hidrocarburos -en 2010 el consumo local de petróleo suponía un $17 \%$ de la producción nacional, mientras que en 2019 el consumo local ya suponía el $41 \%$ de lo producido (Oxford Economics, 2021a)_, lo que podría acabar afectando a la cantidad de petróleo y gas que Argelia podría exportar de forma rentable.

La apuesta por las renovables en estos últimos años en España no ha podido compensar la brecha de dependencia, al coincidir su expansión con el cierre de muchas centrales de carbón. Actualmente, con datos de REE (2020) sobre el mix de generación entre enero y septiembre de 2021, en España la energía eólica produce un $22,2 \%$ del total y la fotovoltaica, un $8,7 \%$, mientras que las centrales de ciclo combinado (gas) producen un $14,9 \%$ y las de carbón, un 1,6\%. La nuclear ocupa aún un lugar destacado, con una producción del 21,7\%, mientras que la hidroeléctrica representa el 12,9\%. Como vemos, la energía nuclear sigue aportando un respaldo importante al mix energético y sigue siendo una pieza clave para mitigar la dependencia de España.

En este marco de volatilidad de precios e incertidumbre sobre la seguridad de suministro europeo, Francia, que obtiene hasta un $70 \%$ de su producción con energía nuclear, está presionando, junto con Polonia, Chequia, Hungría, Rumanía, Eslovaquia y Eslovenia, para que la energía nuclear se incluya dentro de la llamada «taxonomía verde»14, una clasificación que determina a nivel comunitario qué activos pueden ser considerados como «verdes» (cualquier empresa que quiera asegurar que es sostenible frente a sus inversores tendrá que demostrarlo atendiendo a los criterios técnicos especificados en los actos delegados que se derivan del Reglamento 2020/852 de la $U^{15}$ ). De este modo, si la energía nuclear se incluye en la «taxonomía verde», sus activos podrán entrar, por ejemplo, dentro de las inversiones que pueden ser financiadas con bonos verdes, lo que permitirá atraer a un creciente número de inversores (sobre todo, fondos de pensiones y fondos de inversión) dispuestos a aceptar un precio ligeramente más alto a cambio de hacerse con esos títulos.

Además, en la energía nuclear se está produciendo una innovación significativa, que $\triangleright$

\footnotetext{
14 Se espera que la presidenta de la Comisión, Ursula von der Leyen, presente una propuesta a este respecto para que se debata a finales de 2021. La presidenta ha destacado que «es obvio que necesitamos más renovables y energías limpias. Junto a esto, necesitamos una fuente estable: la nuclear. Y durante la transición, por supuesto, gas».

15 El Reglamento 2020/852 sienta las bases para determinar si una actividad económica se considera medioambientalmente sostenible a efectos de fijar el grado de sostenibilidad medioambiental de una inversión. El Reglamento de taxonomía funciona mediante la publicación de actos delegados por parte de la Comisión, que definirán los criterios técnicos para apreciar en qué supuestos se puede considerar que una inversión no causa un perjuicio significativo al medioambiente o que supone una contribución significativa a algunos de los objetivos ambientales.
} 
son los llamados reactores modulares pequeños (SMR, small modular reactors). Estos están siendo desarrollados actualmente por más de setenta compañías alrededor del mundo -entre ellas la británica Rolls-Royce y la estadounidense Terrapower, cofundada por Bill Gates-. Estos reactores son capaces de producir cerca de un tercio de la energía generada por uno tradicional, pero son más fáciles de fabricar en masa y transportar para su ensamblaje. Sus impulsores aseguran que agilizaría la construcción de centrales y rebajaría su coste prohibitivo. Francia anunció recientemente que destinará mil millones de euros al desarrollo de SMR.

Como corolario, cabría señalar que, además de actuar de manera reactiva sobre los retos que plantea la economía poscovid (influencia de la geopolítica sobre el suministro energético, aparición de cuellos de botella, etc.), parece preciso dotarse de instrumentos organizativos para anticipar y prevenir de manera efectiva estos riesgos. Un ejemplo en este sentido es el Estado francés, que para preservar su seguridad económica ha reforzado su organización interna con la creación de un comité de coordinación en materia de seguridad económica. De este modo, el 20 de marzo de 2019, el Gobierno francés publicó un decreto sobre la gobernanza de la política de seguridad económica ${ }^{16}$. Este decreto define el propósito de la política de seguridad económica como la protección y promoción de los intereses económicos, industriales y científicos de la nación, con especial énfasis en los activos estratégicos, ya sean tangibles o intangibles. Se refuerza también la acción colegiada de la política de seguridad económica organizando el trabajo de las Administraciones en torno a un

16 Décret $n^{\circ}$ 2019-206 du 20 mars 2019 relatif à la gouvernance de la politique de sécurité économique. único comité interministerial (el COLISE) y favoreciendo la puesta en común de la información estratégica entre los distintos actores implicados para garantizar la eficacia del sistema en su conjunto.

Por tanto, en el contexto actual de incertidumbre y de desmedida influencia de la geopolítica sobre la economía, parece necesario dotarse de instrumentos como el señalado anteriormente para limitar los riesgos y, sobre todo, tomar decisiones lo más informadas posible, ya sea en el ámbito energético o en otros.

\section{Bibliografía}

Aparicio, L. (25 de octubre de 2021). Los precios energéticos no dan tregua. El País. https://elpais. com/economia/negocios/2021-10-25/los-precios-energeticos-no-dan-tregua.html

BP. (2021). Statistical Review of World Energy 2021, 70th edition. https://www.bp.com/content/dam/ bp/business-sites/en/global/corporate/pdfs/ energy-economics/statistical-review/bp-stats-review-2021-full-report.pdf

CORES. (2021). Estadísticas. https://www.cores.es/ es/estadisticas

Décret n 2019-206 du 20 mars 2019 relatif à la gouvernance de la politique de sécurité économique. Journal Officiel de la République Française, no 0068 du 21 mars 2019, texte 9. https://www.legifrance.gouv.fr/loda/id/JORFTEXT000038252109/

Don't expect big oil to fix the energy crunch (2021, October 16). The Economist. https://www.economist.com/business/2021/10/16/dont-expect-bigoil-to-fix-the-energy-crunch

El déficit de gas natural desencadena una lucha por el suministro antes del invierno (8 de octubre de 2021). World Energy Trade. https://www.worldenergytrade.com/finanzas-energia/economia/ el-deficit-de-gas-natural-desencadena-una-lucha-por-el-suministro-antes-del-invierno 
Enagás. (2021). Boletín Estadístico. Octubre 2021. https://www.enagas.es/stfls/ENAGAS/Gesti \%C3\%B3n \%20T\%C3\%A9cnica \%20del \%20 Sistema/Documentos/DEMANDA/Bolet $\%$ C3 \%ADn \%20Estad \%C3\%ADstico_octubre21.pdf

Escribano, G. (2014). La seguridad energética española en un escenario en transición. Cuadernos de Estrategia, (166), 93-126. https://dialnet.unirioja.es/servlet/articulo?codigo $=4729875$

Escribano, G. (2019). La energía como vector de cooperación y desarrollo sostenible en el Mediterráneo. Revista de Occidente,(461), 23-33.

Escribano, G. (24 de septiembre de 2021). H2 Med: una senda geopolítica de transición para el gas en Europa. Blog Elcano. https://blog.realinstitutoelcano.org/h2-med-una-senda-geopoliticade-transicion-para-el-gas-en-europa/

Escribano, G. (2021). H2 Med: impulsores y barreras geopolíticos y geoeconómicos para el hidrógeno en el Mediterráneo (Elcano Policy Paper, mayo 2021). Real Instituto Elcano. http://www. realinstitutoelcano.org/wps/wcm/connect/ fb2f47cc-bec8-45a4-96be-2820556f363c/PolicyPaper-Escribano-H2-Med-impulsores-barrerasgeopoliticas-geoeconomicas-para-hidrogenoen-Mediterrane.pdf?MOD=AJPERES\&CACHEID= fb2f47cc-bec8-45a4-96be-2820556f363c

Escribano, G., Aires, L., Araluce, I., y Espí Rodríguez, J. A. (2018). Los actores críticos en la nueva geopolítica energética. Executive Excellence: la revista de liderazgo, la gestión y la toma de decisiones, (145), 50-56.

Eurostat. (2021). Energy Data. Comisión Europea. https://ec.europa.eu/eurostat/web/energy/data

Fernández-Cuesta, N. (13 de octubre de 2021). La crisis del gas: el invierno del descontento. El Confidencial. https://blogs.elconfidencial.com/ espana/por-si-acaso/2021-10-13/crisis-gas-inviernodescontento_3305314/

Foulis, P. (2021, November 8). The World Ahead 2022. Energy investment needs to increase - so bills and taxes must rise. The Economist. https://www.economist.com/the-world-ahead/2021/11/08/ energy-investment-needs-to-increase-so-billsand-taxes-must-rise

International Energy Agency. (2020). World Energy Investment 2020. Fuel supply. https://www.iea. org/reports/world-energy-investment-2020/ fuel-supply

Mibgas. (2020). Informe anual del Mercado Organizado de Gas. https://www.mibgas.es/sites/ default/files/mibgas_2020_informe_anual_2.pdf

Moreno García-Cano, L. O. (2017). El sector de la energía en Marruecos: oportunidades estratégicas para España. Boletín Económico de ICE, (3084), 47-55. https://doi.org/10.32796/ bice.2017.3084.5617

Natural-gas prices are spiking around the world (2021, September 25). The Economist. https:// www.economist.com/finance-and-economics/ natural-gas-prices-are-spiking-around-theworld/21804953

Offshore. (2021). Spending recovery still at risk in 2021. Endeavor Business Media. https://www. offshore-mag.com/home/article/14187947/ ep-spending-recovery-still-at-risk-in-2021

OMIE. (2020). Evolución del mercado de electricidad. Informe Anual. https://www.omie.es/sites/ default/files/2021-10/informe_anual_2020_ es.pdf

Oxford Economics. (2021a). Country Economic Forecast Algeria. https://www.oxfordeconomics. com/my-oxford/publications/660178?utm_source= halo

Oxford Economics. (2021b). Natural gas prices surge fivefold, with little relief in sight. Research Briefing. Commodities. https://www.oxfordeconomics.com/my-oxford/publications/652656

Pisani-Ferry, J. (2021a). 21-20 Climate Policy is Macroeconomic Policy, and the Implications Will Be Significant. Peterson Institute for International Economic. https://www.piie.com/system/files/documents/pb21-20.pdf 


\section{LA GEOPOLÍTICA DEL GAS: EL MAGREB Y EL SUMINISTRO ENERGÉTICO EN ESPAÑA}

Pisani-Ferry, J. (2021b). The geopolitical conquest of economics. Bruegel. https://www.bruegel.org/ 2021/10the-geopolitical-conquest-of-economics/

REE. (2019). Interconexiones eléctricas. https:// www.ree.es/sites/default/files/11_PUBLICACIONES/Documentos/06_Interconexiones_DIGITAL.pdf

REE. (2020). El Sistema Eléctrico Español en 2020. https://www.ree.es/sites/default/files/publication/2021/06/downloadable/inf_sis_elec_ ree_2020_0.pdf

Reglamento (UE) 2020/852 del Parlamento Europeo y del Consejo, de 18 de junio de 2020, relativo al establecimiento de un marco para facilitar las inversiones sostenibles y por el que se modifica el Reglamento (UE) 2019/2088. Diario
Oficial de la Unión Europea, L 198, de 22 de junio de 2020, pp. 13 a 43. https://eur-lex.europa. eu/legal-content/ES/TXT/PDF/? uri=CELEX: 32020R0852

Sedigas. (2021). Estadísticas. https://www.estadisticasdelgas.es/index

The first big energy shock of the green era (2021, October 16). The Economist. https://www. economist.com/leaders/2021/10/16/ the-first-big-energy-shock-of-the-green-era

Ukrinform. (16 de septiembre de 2020). Ucrania recibirá alrededor de 2.000 millones de dólares por tránsito de gas desde Rusia. https://www. ukrinform.es/rubric-economy/3100552-kobolyevucrania-recibira-alrededor-de-2-mil-millones-portransito-de-gas-desde-rusia.html 
